\title{
El desayuno escolar; una intervención educativa en alimentación y nutrición saludable ${ }^{1^{*}}$
}

\section{School breakfast, an educative intervention in healthy food and nutrition}

\section{Javier Cubero ${ }^{(1)}$, Lourdes Franco-Reynolds ${ }^{(1)}$, Manuel A. Calderón ${ }^{(1)}$, Berta Caro $^{(2)}$, Maximiliano Rodrigo ${ }^{(3)}$, Constantino Ruiz ${ }^{(1)}$}

(1) Laboratorio de Educación para la Salud. Área de Didáctica de Ciencias Experimentales. (2) Área de Fisioterapia. Universidad de Extremadura. Badajoz. Campus de Excelencia HIDRANATURA.

(3) Dpto. de Didáctica de Ciencias Experimentales. Universidad Complutense de Madrid.

Resumen: El Desayuno Completo y Saludable es básico para el crecimiento además favorece un mayor rendimiento físico e intelectual durante la mañana. Dicho Desayuno debe ser siempre: Lácteos, Cereales, Frutas y sus derivados, sin olvidarnos de nuestro Aceite de Oliva. Saltarse o realizar un desayuno de mala calidad es un factor que, además, origina sobrepeso y obesidad infantil. Por ello las Organizaciones Promotoras de la Salud fomentan la intervención de hábitos saludables en Alimentación y Nutrición. Siendo uno de los objetivos este Desayuno Completo y Saludable el cual desde la escuela debe promocionarse, sobre todo fomentando la ingesta de fruta. En base a lo expuesto nuestro trabajo diseña una intervención escolar para un Desayuno Completo y Saludable, centrada en el consumo de fruta.

Palabras clave: Desayuno, escuela, nutrición, educación y salud.

Abstract: The Complete and Healthy Breakfast is basic for growth also favors greater physical and intellectual performance during the morning. Breakfast should always said: Dairy, Grains, Fruit, and their derivates, without forgetting our olive oil. Or to perform skipping breakfast shoddy is a factor that also causes childhood obesity and overweight. Therefore Organizations Promoting Health intervention encourage healthy habits in Food and Nutrition. Being one of the goals this Complete and Healthy Breakfast from school which should be promoted, especially by encouraging the consumption of fruit. Based on the above our work designs a school intervention for a Complete and Healthy Breakfast, centered on the consumption of fruit.

Keywords: Breakfast, school, nutrition, education \& health.

(Fecha de recepción: enero, 2015, y de aceptación: febrero, 2017)

DOI: 10.7203/DCES.32.4546

${ }^{1}$ Los autores desean mostrar su agradecimiento a los tres profesores universitarios y los docentes de los colegios, pues sin su profesionalidad y colaboración, sobre todo en el mantenimiento del orden y motivación a los alumnos, la actividad realizada no habría sido tan eficaz.

"Esta investigación ha sido financiada por: El Área de Cultura y Deportes de la Diputación de Badajoz 2012. Fondos FEDER Gobierno de Extremadura y Universidad de Extremadura (España). Así agradecer a los diferentes colegios de la Provincia de Badajoz y a sus profesores, la colaboración y el valioso interés mostrado en dicha investigación. 


\section{Introducción}

El Desayuno Escolar; un recurso en Educación para la Salud

El desayuno posee un papel básico en el óptimo desarrollo de las etapas de crecimiento infantil, adolescente y juvenil, asociándose a un mayor rendimiento físico e intelectual y por lo tanto académico (Sánchez y Serra, 2000; Córdoba et al., 2014), por el contario saltarse el desayuno afecta a la memoria reciente, a la atención, a la capacidad de resolver problemas, sin olvidar los problemas emocionales (Vaisman et al., 1996; Wesnes et al., 2003; Galiano y Moreno, 2010).

Estos preocupantes y negativos hábitos en el desayuno fueron recogidos, en nuestro país, en trabajos de investigación como el valioso Estudio Enkid, (Serra y Aranceta, 2000), donde se reveló que entre un 8-9\% de los escolares no desayunan nada y sólo un 25-29\%, realizan un Desayuno Completo y Saludable, es decir de Buena Calidad.

Un Desayuno Completo y Saludable, según indica el Ministerio de Sanidad, Servicios Sociales e Igualdad, a través del Programa Perseo Estrategia NAOS es aquel en el que se ingiere, al comenzar el día, los 3 siguientes grupos de Alimentos:

Cereales,

Lácteos,

Fruta y sus derivados.

Sin olvidarnos del Aceite de Oliva, pilar fundamental de nuestra saludable Dieta Mediterránea (DM).
Debemos indicar como posible consecuencia del desequilibrio alimentario infantil, producido entre otros factores por la carencia de un desayuno de buena calidad nutricional y energética, la preocupante obesidad infantil.

Para el caso particular de nuestra región, Extremadura, aclarar que se encuentra entre los primeros y alarmantes puestos de sobrepeso y obesidad infantil en nuestro país, según indican los últimos resultados del Programa $A L A D I N O$ en 2011, recogidos por la Agencia Española de Seguridad Alimentaria (AESAN).

La organización de nuestro Sistema Educativo establece en diez años (de los seis a los dieciséis) el periodo de obligatoriedad escolar. Este periodo que permite una programación de las diferentes materias en un sentido longitudinal, a la hora de impartir los diferentes contenidos sobre alimentación y nutrición, con las diferentes materias que facilitan la inclusión de los mismos en el horario escolar y en el refuerzo desde diferentes aspectos tal y como recoge el valioso Libro Blanco de la Nutrición en Espa$\tilde{n} a$ en 2013 y en particular la legislación educativa para Educación Primaria (LOE, 2006; LOMCE, 2013). Admitiendo también el tratamiento de estos temas de forma transversal colaborando para ello, de forma sinérgica, personal docente y sanitario (Talavera y Gavidia, 2013).

Todos estos argumentos, parecen más que convincentes, para promocionar de una forma profesional y adecuada la educación alimentaria y nutricional, siendo la infancia el mejor 
momento para instaurar unos hábitos alimentarios saludables, como base de un estilo de vida saludable. Para ello es necesario llevar a cabo acciones de intervención en educación nutricional, en el ámbito escolar (Rodrigo et al., 2012) pero no sólo de forma transversal, ya que hasta ahora y los a vista de los resultados obtenidos en Educación para la Salud (EpS) no han sido satisfactorios, (Cubero et al., 2011).

Resaltar que para conseguir aprendizajes significativos los estudiantes deben conocer sus ideas y hábitos con respecto al tema de la alimentación y nutrición, para que luego, puedan llevar a cabo la reestructuración de los mismos (Nuñez y Banet, 2000). Por todo ello, para que sea efectiva una intervención en educación nutricional, esta debe apoyarse e integrarse de forma longitudinal en las asignaturas con contenidos propios de alimentación, nutrición $y$ salud (Cubero et al., 2011), e higiene (Fernández-Manzano et al., 2014) es decir en los contenidos: conceptuales, procedimentales y actitudinales de los currículum escolares, los cuales servirán para desarrollar competencias básicas como: conocimiento y la interacción con el mundo físico, así como la de autonomía e iniciativa personal. Sin olvidar nunca, dicha promoción e intervención desde el ámbito familiar, de lo contrario estaremos abocados al fracaso.

\section{Justificación}

En base a los estudios previos realizados en diferentes colegios de la pro- vincia de Badajoz sobre el análisis del Desayuno en escolares del tercer ciclo de Educación Primaria (Cubero et al., 2014), los autores podemos destacar que el "Desayuno de BUENA CALIDAD" es decir, aquellos en el que los escolares de nuestra población ingerían los 3 tipos de Alimentos o sus derivados tan sólo alcanza un $32,2 \%$, o lo que es lo mismo, un $67,8 \%$ no desayunaba de forma completa y saludable, por ello creemos conveniente y más que recomendable, llevar a cabo una intervención educativa en alimentación y nutrición saludable, concretamente en la primera comida del día: el Desayuno.

Conforme a los resultados de la calidad del Desayuno que se desprenden del estudio Enkid 2000, queremos destacar cómo el "Desayuno de MEJORABLE CALIDAD” es el más común entre los escolares, siendo el grupo de las Frutas y sus derivados el gran ausente en este desayuno, hecho que ocurre de igual forma con este grupo de alimento en nuestra población analizada (Cubero et al., 2014). Por ello, es necesario realizar una correcta intervención educativa para impulsar el consumo de fruta en el desayuno, sin olvidar los otros dos grandes grupos de alimentos básicos.

\section{Intervención Educativa en Alimentación y Nutrición: $E l$ Desayuno Completo y Saludable}

Ésta fue diseñada e impartida por los autores, se desarrolló en el marco de las Jornadas: Desayunos con la Ciencia, organizado por el Servicio de Divul- 
gación Científica de la Universidad de Extremadura, durante el curso 20132014, y cuyo objetivo prioritario es acercar la Ciencia a la comunidad escolar.

Dicha Intervención sobre el Desayuno Completo y Saludable estuvo dirigida a grupos de 25 alumnos/as de $3^{\circ}$ Ciclo de Educación Primaria, con rango de edad 10-12 años, de diferentes colegios de la Provincia de Badajoz.

La intervención, con una duración aproximada de 1 hora, fue llevada a cabo por 3 profesores universitarios, acompañados de los docentes de estos centros educativos. No obstante, debemos aclarar que esta intervención está diseñada para poder ser realizar por un único docente aunque la duración de la misma, siempre, superará la hora de duración.

Dicha intervención se dividió en 4 Fases:

$1^{\circ}$ - En la Primera Fase, se evaluó mediante -Cuestionario Recordatorio de 24h (ANEXOS)- (es decir preguntas sobre el consumo de Lácteos, Cereales y Frutas y sus derivados en el desayuno y a media mañana de ese día) diseñado y realizado para estos escolares por expertos en nutrición y educación, añadir que también fueron evaluadas las emociones (positiva o negativas) frente a dicho desayuno. La duración de la misma fue de 15 minutos.

$2^{\circ}$ - En la Segunda Fase, se impartieron contenidos básicos y elementales sobre el porqué del Desayuno Completo $y$ Saludable y qué alimentos deben constituirlo. Para ello nos apoyamos en una 4 láminas diseñada por los autores
(ANEXOS), donde se enseñan dicho contenidos. La duración fue de 15 minutos. $3^{\circ}$ - En la $3^{\mathrm{a}}$ Fase evaluamos dicho Cuestionario Recordatorio de 24h, de forma individual por cada profesor y posteriormente de forma colectiva, centrándonos en el grupo de Alimento que consumía en menor porcentaje, como era de suponer en base ya a trabajos previos ya realizados por los autores (Cubero et al., 2014), fue el de la Fruta y sus derivados.

$4^{\circ}$-En la $4^{\text {a }}$ Fase y última, los escolares eran invitados al consumo de alguna pieza de fruta fresca, la cual era expuesta de forma atractiva para este fin a través del Plato Saludable de Fruta. Recordar que tras este aprendizaje el escolar consumió fruta de forma satisfactoria y un elevadísimos porcentaje interiorizó el beneficio de Desayuno Completo y Saludable, gracias sobre todo a la promoción de este consumo de fruta fresca.

Por ello los profesores involucrados, para cerciorarnos de dicha correcta enseñanza previamente diseñamos y elaboramos una Rúbrica de autoevaluación (Martínez-Figueira et al, 2013), en la cual se analizaría el aprendizaje a este grupo de escolares.

Dicha Rubrica analítica (MartínezRojas 2008) está centrada en cada una de las 4 Fases descritas en la intervención educativa y mediante la cual se analizó el aprendizaje de los conocimientos transmitidos en 7 Criterios. A partir de estos Criterios ya establecidos, se elaboró un cuadro con los descriptores de cada una de las categorías o aspectos 
evaluados, organizando el cálculo de la calificación con esta rúbrica si se quisiese dar una nota Global.

Aclarar que cada uno de estos 7 Criterios, eran calificados en base a 4
Categorías con la siguiente puntuación: Excelente: 4 ptos. Bien: 3 ptos. Regular: 2 ptos y Deficiente: 1 pto. Existiendo un Rango de Calificación de 28-0. Siendo el valor Medio para 14 ptos.

\section{Cuadro I}

Rúbrica de autoevaluación para los docentes para Intervención Educativa para el Desayuno Completo y Saludable

Fuente: Elaboración propia.

\begin{tabular}{|c|c|c|c|c|c|}
\hline Criterios & $\begin{array}{l}\text { Excelente } \\
4 \text { ptos }\end{array}$ & $\begin{array}{c}\text { Bien } \\
3 \text { ptos }\end{array}$ & $\begin{array}{c}\text { Regular } \\
2 \text { ptos }\end{array}$ & $\begin{array}{c}\text { Deficiente } \\
1 \text { pto }\end{array}$ & $\begin{array}{c}\text { Total } \\
\text { ptos }\end{array}$ \\
\hline \multicolumn{6}{|l|}{$\mathbf{1}^{\mathbf{a}}$ Fase } \\
\hline \multicolumn{6}{|l|}{$\begin{array}{l}\text {-Contestaron al cuestionario- } \\
\text { recordatorio sin dificultad en el } \\
\text { tiempo estimado para ello }\end{array}$} \\
\hline \multicolumn{6}{|l|}{$2^{\text {a }}$ Fase } \\
\hline \multicolumn{6}{|l|}{$\begin{array}{l}\text {-Comprendieron los contenidos } \\
\text { básicos y elementales sobre el } \\
\text { Desayuno Completo y Saludable }\end{array}$} \\
\hline \multicolumn{6}{|l|}{$\begin{array}{l}\text {-Identifican correctamente los } \\
\text { alimentos que componen el } \\
\text { Desayuno Completo y Saludable }\end{array}$} \\
\hline \multicolumn{6}{|l|}{$3^{a}$ Fase } \\
\hline \multicolumn{6}{|l|}{$\begin{array}{l}\text {-La ingesta de lácteos, cereales y } \\
\text { fruta analizados es adecuada }\end{array}$} \\
\hline \multicolumn{6}{|l|}{$\begin{array}{l}\text {-Consumen de forma habitual } \\
\text { fruta y sus derivados en el } \\
\text { Desayuno }\end{array}$} \\
\hline \multicolumn{6}{|l|}{$4^{\text {a }}$ Fase } \\
\hline \multicolumn{6}{|l|}{$\begin{array}{l}\text {-Se acercan a los platos } \\
\text { saludables de fruta expuestos } \\
\text { para ellos }\end{array}$} \\
\hline $\begin{array}{l}\text {-Consumieron fruta de forma } \\
\text { satisfactoria y aprendieron sus } \\
\text { beneficios }\end{array}$ & & & & & \\
\hline
\end{tabular}


Resultados

Referente a los resultados obtenidos, del análisis del aprendizaje a partir de la Rúbrica de autoevaluación de los docentes (Tabla I), principalmente, destacaremos que tras el cálculo de la puntuación de los 7 Criterios, en base a la 4 Categorías, la Nota Global obtenida fue el valor medio de 23,32 sobre un máximo de 28 ptos, lo que significa que en un $83,28 \%$ de los Criterios su puntuación elegida fue la máxima, correspondiendo a 4 ptos.

\section{TABLA I. Puntuación por Rúbrica de autoevaluación de los docentes para Intervención Educativa para el Desayuno Completo y Saludable.}

\begin{tabular}{|l|c|}
\hline Criterios & $\begin{array}{c}\text { Puntuación } \\
\text { Media }\end{array}$ \\
\hline $\mathbf{1}^{\mathbf{a}}$ Fase & 3,66 \\
\hline $\begin{array}{l}\text {-Contestaron al cuestionario-recordatorio sin dificultad en el } \\
\text { tiempo estimado para ello }\end{array}$ & 3,66 \\
\hline $\mathbf{2}^{\mathbf{a}}$ Fase & 4 \\
\hline $\begin{array}{l}\text {-Comprendieron los contenidos básicos y elementales sobre el } \\
\text { Desayuno Completo y Saludable }\end{array}$ & \\
\hline $\begin{array}{l}\text {-Identifican correctamente los alimentos que componen el } \\
\text { Desayuno Completo y Saludable }\end{array}$ & 2 \\
\hline $\mathbf{3}^{\mathbf{a}}$ Fase & 2 \\
\hline -La ingesta de lácteos, cereales y fruta analizados es adecuada \\
\hline -Consumen de forma habitual fruta y sus derivados en el Desayuno & 4 \\
\hline $\mathbf{4}^{\mathbf{a}}$ Fase & 4 \\
\hline -Se acercan a los platos saludables de fruta expuestos para ellos & \\
\hline $\begin{array}{l}\text {-Consumieron fruta de forma satisfactoria y aprendieron sus } \\
\text { beneficios }\end{array}$ & 23,32 \\
\hline Nota Global & \\
\hline
\end{tabular}

Con respecto a la Categoría destacada por los docentes frente a estos 7 Criterios (Tabla II), reseñar que fue la de Excelente, coincidiendo en un $62 \%$ de los casos. Aclarar en particular, que para los Criterios de la Fase 3 centrada en analizar la ingesta del desayuno de estos escolares, en su totalidad los docentes coincidía con la Categoría de Regular. Mientras que para la Categoría de Bien se dio tan solo en un $9,5 \%$ de los casos, en particular para el Criterio 1 de la Fase 1; ya que no contestaron al cuestionario-recordatorio en el tiempo 
estimado para ello y para el Criterio 2 de la Fase 2 ya que no identifican correc- tamente los alimentos que componen el Desayuno Completo y Saludable.

\section{TABLA II. \% de las Categorías de la Rúbrica de autoevaluación de los docentes para Intervención Educativa para el Desayuno Completo y Saludable}

\begin{tabular}{|c|c|c|c|c|}
\hline Categorías & Excelente & Bien & Regular & Deficiente \\
\hline$\%$ & $62 \%$ & $9,5 \%$ & $28,5 \%$ & $0 \%$ \\
\hline
\end{tabular}

\section{Discusión y conclusión}

En base a todo lo expuesto hasta ahora, sería muy recomendable que esta intervención fuera realizada dentro de los centros educativos apoyándose en el proyecto curricular dentro de la Asignatura de Conocimiento del Medio (otra acertada opción sería también dentro de la asignatura de Educación Física) en el tercer ciclo de Educación Primaria. Se recomienda que estas intervenciones en Educación para la Salud, siempre sean enmarcadas dentro de contenidos longitudinales de: alimentación, nutrición y salud ya que las leyes educativas actuales, afortunadamente, así lo contemplan. Esta intervención en EpS no será dejada caer, nunca mejor dicho, en la transversalidad de contenidos, porque tal y como todos sabemos sus resultados estarán abocados al fracaso, o lo que es peor no serán realizadas (Cubero et al., 2011).

A través de reuniones se informaría a los padres/tutores, y con su consentimiento, se les pediría su colaboración en dicha proceso de enseñanza. Sin olvidar la recomendable alianza del docente con el profesional socio-sanitario, consiguiendo así que nuestra promoción en salud, través de la EpS, esté acreditada y garantizada por sus tres pilares fundamentales: familia, personal docente y sanitario (Talavera y Gavidia, 2013).

La intervención se dividirá en tres Fases, de un mes de duración cada una:

$1^{\circ}$ - En la Primera Fase, durante el primer mes, se realizarían tres Sesiones en semanas consecutivas, con un tema específico por sesión:

- $1^{\mathrm{a}}$ sesión. Desayuno Completo y Saludable

- $2^{\text {a }}$ sesión: 3 raciones de fruta al día

- $3^{\mathrm{a}}$ sesión: Desayuno en familia y Evaluación.

Dentro de la Asignatura de -Conocimiento del Medio- y aprovechando la enseñanza de contenidos conceptuales de alimentación, nutrición y promoción de la salud (Cubero et al., 2012), se dará a conocer a los escolares la importancia de la primera comida del día, así como los beneficios que conlleva la ingesta de Fruta y sus derivados y cómo introdu- 
cirla en la dieta diaria, con un mínimo de 3 raciones (80-125g) diarias.

$2^{\text {a }}$ - La Segunda Fase, abordada en el segundo mes, sería totalmente práctica o procedimental. En ella, el grupo clase elaboraría un mural, apoyándonos en el modelo explicado en pizarra (digital o tradicional), que llevaría como título "Desayuno Completo y Saludable", donde se destacaría los tres grandes grupos de alimentos del Desayuno (Lácteos, Cereales, Fruta y sus derivados, sin olvidarnos de nuestro Aceite de Oliva), así como la importancia de realizar este Desayuno en la mejora del rendimiento escolar (Kleinman et al., 2002; Córdoba et al., 2014).

Otra actividad práctica, dentro de esta Segunda Fase, consistiría en realizar un Taller en el comedor del colegio, en el que se prepararían "Platos Saludables de Fruta" para tomarlas a media mañana, durante el recreo. Ya que así la fruta resulta más atractiva y su ingesta resulta más recreativa. A partir de un cada alumno diseñaría y combinaría a su gusto los trozos de frutas en colaboración con la nutricionista del colegio, dándoles así la libertad de poder elegir sus sabores preferidos.

La última actividad práctica de esta Segunda Fase se desarrollaría también en el comedor del colegio, si es posible con la colaboración de las familias. Y donde cada uno de los escolares elaboraría su propio desayuno.

En el comedor se expondrían diferentes alimentos, saludable y no saludables (bollería, golosinas...), para que ellos mismo eligieran los más ade- cuados para su Desayuno Completo y Saludable, teniendo en cuenta todo lo aprendido en la primera fase. Ni que decir tiene, dado que esta intervención queda refrendada dentro de los contenidos longitudinales de alimentación, nutrición y salud, que su aprendizaje quedará evaluado a través de las mismas pruebas de contenidos dentro de la asignatura oficialmente impartida de Conocimiento del Medio (otra acertada opción sería también dentro de la asignatura de Educación Física).

$3^{\mathrm{a}}$ - La Tercera y última Fase consistiría únicamente en dos Sesiones, a realizar durante el tercer y último mes de la intervención:

En la Primera Sesión, serían los propios escolares los que transmitirían mediante aprendizaje cooperativo (Gallach et al., 2014) a los compañeros de otro curso la importancia de consumir fruta en el desayuno, acompañada además de lácteos, cereales y sus derivados, así como también la ingesta de fruta a media mañana. (Utilizarían para ello el gran mural desarrollado en la primera fase).

Y la Segunda y por tanto última Sesión sería dedicada a la revisión y aplicación de todo lo aprendido durante las Tres Fases desarrolladas, mediante un debate en el aula con el grupo-clase. Y para finalizar y no menos importante, una Evaluación mediante una prueba escrita donde deban explicar: cómo tienen que desayunar en casa antes de acudir al colegio, por qué es importante realizarlo de manera saludable, aportar alguna propuesta para estar bien ali- 
mentado a media mañana y argumentar los beneficios de la ingesta de fruta en el Desayuno. Por último, no podemos olvidar la recomendación a los docentes de utilizar la Rúbrica aquí manejada (Martínez-Figueira et al, 2013), como herramienta de autoevaluación de sus enseñanzas de Intervención Educativa para el "Desayuno Completo y Saludable".

En conclusión se debe promocionar de una forma profesional y adecuada la educación alimentaria y nutricional, siendo la infancia el mejor momento para instaurar unos hábitos alimentarios saludables. Para ello es necesario llevar a cabo en el ámbito escolar, y través de la EpS, acciones de intervención en educación nutricional a un nivel curricular longitudinal además del transversal, revirtiendo en un beneficio del rendimiento académico como ocurre con el "Desayuno Completo y Saludable", además de establecer un estilo de vida saludable.

\section{Anexo. \\ Imagen de un ejemplo de Cuestionario \\ Recordatorio sobre el desayuno, completado por la población escolar en la Universidad de Extremadura.}

Fuente: Elaboración propia.
ENCUESTA SOBRE HÁBITOS ALIMENTARIOS: DESAYUNO.

SEXO: EDAD: PESO: ALTURA: 1,54

1.- Desayunas: si $\square$ No $\square$

2.- ¿Cómo desayunas?
$\square$ solo.
\ Acompañado de algún familiar

3.- ¿Cuánto tiempo dedicas al desayuno?

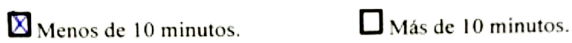

4.- ¿Qué haces mientras desayunas?
$\square$ Sólo desayuno.
冈eo la televisión.
区 Hablo con alguien.
$\square$ Juego.
Hago las tareas.
Qotras cosas

5,- ¿Qué has desayunado hoy?

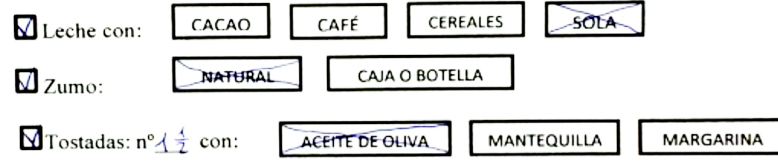

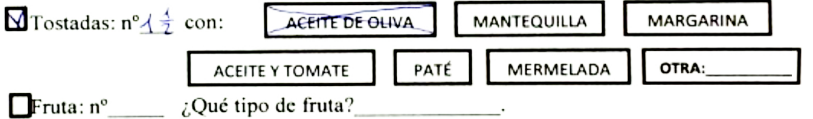

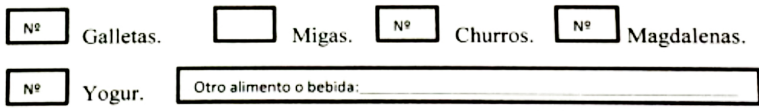

6.- El desayuno de hoy, ¿es igual casi todos los días? SI $\square$ No $\square$

7,-iLlevas comida o bebida al colegio para tomar en el recreo? SI $\square$ No

8.- En caso de llevarla, ¿qué has traido hoy?

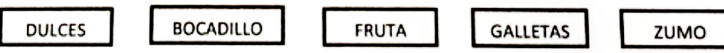

9.- Si no la llevas este curso, ¿la has llevado en anteriores? SI $\square$ No $\square$ 


\section{Referencias bibliográficas}

CÓRDOBA, L., LUENGO, L.M. y GARCÍA, V. (2014). Análisis de los conocimientos sobre el desayuno saludable y su relación con los hábitos de estilo de vida y el rendimiento académico en la enseñanza secundaria obligatoria. Endocrinología $y$ Nutrición, 61(5), 242-251. DOI: 10.1016/j.endo-nu.2013.11.006.

CUBERO, J., CALDERÓN, M., COSTILLO, E., CAÑADA, F. y RUIZ, C. (2011). La Educación para la Salud en el Espacio Europeo de Educación Superior. Publicaciones, 41, 51-64.

CUBERO, J., CALDERÓN, M., POZO, A. y RUIZ, C. (2013). Análisis de la calidad del desayuno en una población escolar de Badajoz. Revista Campo Abierto, 32(2), 145-154.

CUBERO, J., CAÑADA, F., COSTILLO, E., FRANCO, L., CALDERÓN, A., SANTOS, AL., PADEZ, C. y RUIZ, C. (2012). La alimentación preescolar, educación para la salud de los 2 a los 6 años. Enfermería Global, 11(3), 337-345. DOI: 10.4321/ S1695-61412012000300018.

CUBERO, J., GUERRA, S., CALDERÓN, M., LUENGO, M., POZO, A. y RUIZ, C. (2014). Análisis del desayuno escolar en la provincia de Badajoz. Revista Española de Nutrición Comunitaria, 20(2), 51-56. DOI: 10.7400/RENC.2014.01.2.5011

Desayunos Saludables Campaña Escolar 2011-2012. Comunidad de Madrid. 1-37.
FERNANDEZ-MANZANO, L., TALAVERA, M., FURIO, C. y GAVIDIA, V. (2014). La higiene en el currículo escolar de la Educación Obligatoria española. Didácticas de las Ciencias Experimentales y Sociales, 28, 243-262. DOI: 10.7203/dces.28.4173.

GALIANO, MJ. y MORENO, JM. (2010). El desayuno en la infancia: más que una buena costumbre. Acta Pediátrica Española, 68(8), 403-408.

GALLACH, MJ. y CATALAN, J.P. (2014). El aprendizaje Cooperativo en Primaria: Teoría, Práctica y Actividades Concretizadas. Didáctica de las Ciencias Experimentales y Sociales, 28, 109-133. DOI: 10.7203/dces.28.3810.

KLEINMAN, R.E., HALL, S., GREEN, H., KORZEC-RAMIREZ, D., PATTON, K., PAGANO, M.E. y MURPHY,J.M.(2002). Diet, breakfast, and academic performance in children. Annals Nutrition Metabolism, 46(1), 24-30. DOI: 10.1159/000066399.

Ley Orgánica 2/2006, de 3 de mayo, de Educación (LOE). Boletín Oficial del Estado (España), 4 de mayo de 2006, 106, 17158-17207.

Ley Orgánica 8/2013, de 9 de diciembre, para la Mejora da Calidad Educativa. Boletín Oficial del Estado (España), 10 de diciembre de 2013, 295, 97858-97921.

MARTÍNEZ, J.R. (2014). Guía fácil para un desayuno y una merienda saludable. Fundación Alimentación Saludable. Sociedad Española de 
Dietética y Ciencias de la Alimentación (SEDCA).

MARTINEZ-ROJAS, JM. (2008). La rúbrica en la evaluación escolar: su construcción y uso. Avances en medición, 6, 129-138.

MARTINEZ-FIGUEIRA, E., TELLADO-GONZALEZ, F., RAPOSORIVAS, M. (2013). La rúbrica como instrumento de autoevaluación: un estudio piloto. Revista de Docencia Universitaria, 12(2), 373-390.

Ministerio de Educación Cultura y Deporte (2014). Real Decreto $126 / 2014$, de 28 de febrero, por el que se establece el currículo básico de la Educación Primaria. Boletín Oficial del Estado (España), 1 de marzo de 2014, 52, pp. 19349-19420.

Ministerio de Sanidad y Consumo (2007). "Empezar el día con un desayuno saludable”. Programa PERSEO. Estrategia para la Nutrición, Actividad Física y Prevención de la Obesidad (NAOS). España: Agencia Española de Seguridad Alimentaria y Nutrición (AESAN).

Ministerio de Sanidad, Servicios Sociales e Igualdad (2011). "Estudio de prevalencia de la obesidad infantil”. Estudio ALADINO. Estrategia para la Nutrición, Actividad Física y Prevención de la Obesidad (NAOS). España: Agencia Española de Seguridad Alimentaria y Nutrición (AESAN).

NÚÑEZ, F. y BANET, E. (2000). Aprender sobre la alimentación para desa- rrollar hábitos y actitudes saludables en el alumnado de primaria. Aula de Innovación Educativa, 92, 9-14.

RODRIGO, M., EJEDA, J.M. y CABALLERO, M. (2012). Una década enseñando e investigando en Educación Alimentaria para Maestros. Revista Complutense en Educación, 24(2), 243-265. DOI: 10.5209/rev_ rced.2013.v24.n2.42078.

RODRIGO, M., IGLESIAS, M.T., EJEDA, J.M., CABALLERO, M. y ORTEGA, M.C. (2014). Una investigación en torno al desayuno realizado por futuros maestros y algunas propuestas para la mejora de la educación alimentaria. Nutrición Clínica y Dietética Hospitalaria. En prensa.

SÁNCHEZ, J. y SERRA, L. (2000). Importancia del desayuno en el rendimiento intelectual y en el estado nutricional de los escolares. Revista Española de Nutrición Comunitaria, 6, 53-95.

SERRA, L. y ARANCETA, J. (2000). Desayuno y Equilibrio Alimentario. Estudio Enkid. Madrid: Masson.

TALAVERA, M. y GAVIDIA, V. (2013). Percepción de la educación para la salud en el personal docente y sanitario. Didáctica de las Ciencias Experimentales y Sociales, 27, 115129. DOI: 10.7203/dces.27.2569.

VAISMAN, N., VOET, H., AKINVIS, A. y VAKIL, E. (1996). The effects of breakfast timing on the cognitive fuction of elementary school stu- 
dents. Archive Pediatric Adolescent Medical, 150(10), 1.089-1.092.

VARELA, G. (coord.) (2013). El Libro Blanco de la Nutrición en España. Madrid: Fundación Española de la Nutrición.
WESNES, K. A. y PINCOCK, C., RICHARDSON, D., HELM, G. y HAILS, S. (2003). Breakfast reduces declines in attention and memory over the morning in schoolchildren. Appetite, 41(3), 329-331. DOI: 10.1016/j.appet.2003.08.009. 